\title{
Digital Filtering Performance in the ATLAS Level-1 Calorimeter Trigger
}

\author{
David R. Hadley on behalf of the ATLAS collaboration
}

\begin{abstract}
The ATLAS Level-1 Calorimeter Trigger is a hardware-based system designed to identify high- $p_{T}$ jets, electron/photon and tau candidates, and to measure total and missing $E_{T}$ in the ATLAS Liquid Argon and Tile calorimeters. It is a pipelined processor system, with a new set of inputs being evaluated every $25 \mathrm{~ns}$. The overall trigger decision has a latency budget of $\sim 2 \mu \mathrm{s}$, including all transmission delays. The calorimeter trigger uses about 7200 reduced-granularity analogue signals, which are first digitized at the $40 \mathrm{MHz}$ LHC bunchcrossing frequency, before being passed to a digital Finite Impulse Response (FIR) filter. Due to latency and chip real-estate constraints, only a simple 5-element filter with limited precision can be used. Nevertheless, this filter achieves a significant reduction in noise, along with improving the bunch-crossing assignment and energy resolution for small signals. The context in which digital filters are used for the ATLAS Level-1 Calorimeter Trigger is presented, before describing the methods used to determine the best filter coefficients for each detector element. The performance of these filters is investigated with commissioning data and crosschecks of the calibration with initial beam data from ATLAS are shown.
\end{abstract}

\section{INTRODUCTION}

$\mathbf{T}$ HE ATLAS trigger consists of three levels. Level-1 systems are hardware-based and operate with a fixed latency of $\sim 2 \mu \mathrm{s}$. The Level-2 trigger is software-based with a mean-processing time of $40 \mathrm{~ms}$ per event; its algorithms are seeded by regions-of-interest identified by the first level

Manuscript received May 28, 2010. We acknowledge the support of ANPCyT, Argentina; Yerevan Physics Institute, Armenia; ARC and DEST, Australia; Bundesministerium für Wissenschaft und Forschung, Austria; National Academy of Sciences of Azerbaijan; State Committee on Science \& Technologies of the Republic of Belarus; CNPq and FINEP, Brazil; NSERC, NRC, and CFI, Canada; CERN; CONICYT, Chile; NSFC, China; COLCIENCIAS, Colombia; Ministry of Education, Youth and Sports of the Czech Republic, Ministry of Industry and Trade of the Czech Republic, and Committee for Collaboration of the Czech Republic with CERN; Danish Natural Science Research Council and the Lundbeck Foundation; European Commission, through the ARTEMIS Research Training Network; IN2P3-CNRS and CEADSM/IRFU, France; Georgian Academy of Sciences; BMBF, DFG, HGF and MPG, Germany; Ministry of Education and Religion, through the EPEAEK program PYTHAGORAS II and GSRT, Greece; ISF, MINERVA, GIF, DIP, and Benoziyo Center, Israel; INFN, Italy; MEXT, Japan; CNRST, Morocco; FOM and NWO, Netherlands; The Research Council of Norway; Ministry of Science and Higher Education, Poland; GRICES and FCT, Portugal; Ministry of Education and Research, Romania; Ministry of Education and Science of the Russian Federation and State Atomic Energy Corporation ROSATOM; JINR; Ministry of Science, Serbia; Department of International Science and Technology Cooperation, Ministry of Education of the Slovak Republic; Slovenian Research Agency, Ministry of Higher Education, Science and Technology, Slovenia; Ministerio de Educación y Ciencia, Spain; The Swedish Research Council, The Knut and Alice Wallenberg Foundation, Sweden; State Secretariat for Education and Science, Swiss National Science Foundation, and Cantons of Bern and Geneva, Switzerland; National Science Council, Taiwan; TAEK, Turkey; The Science and Technology Facilities Council and The Leverhulme Trust, United Kingdom; DOE and NSF, United States of America. trigger. Finally, the Event Filter uses the full detector information to reconstruct events with a mean processing time of $4 \mathrm{~s}$. The Level-1 Trigger consists of three main systems: the Calorimeter Trigger, which uses reduced-granularity information from the calorimeters to search for high- $E_{\mathrm{T}}$ objects; the Muon Trigger, which uses specialised detectors to identify high- $p_{\mathrm{T}}$ muons; and the Central Trigger Processor, where the final Level-1 trigger decision is made.

The Level-1 Calorimeter Trigger (L1Calo) achieves the required density and speed of algorithmic processing through the use of FPGAs and ASICs. The real-time path in the trigger is subdivided into three stages. The Preprocessor, which receives and digitizes analogue signals from the calorimeters, is followed by two digital processor systems working in parallel, the Jet/Energy-Sum Processor and the Cluster Processor. L1Calo provides all of the calorimeter-based trigger information used by the Central Trigger Processor, providing the majority of the individual inputs to the overall Level-1 trigger decision.

The Level-1 trigger decision has to be distributed to the detector front-end systems in $\sim 2 \mu \mathrm{s}$. After subtracting the cable delays, the L1Calo system itself has a latency budget of $\sim 1 \mu \mathrm{s}$, of which the Preprocessor uses less than $400 \mathrm{~ns}, 16$ LHC bunch-crossing periods.

The Preprocessor internally consists of several stages, with the majority of the processing performed on a dense MultiChip Module consisting of FADCs, a single ASIC, and serialized output-driver chips. The heart of the system is the ASIC, a pipelined digital processing element, which converts the digitized input signals into correctly time-aligned, calibrated and noise-suppressed outputs. The assignment of the energy deposits to the correct LHC bunch-crossing is referred to as Bunch-Crossing Identification (BCID). The energy resolution has to be of the order of $3 \%$ of the calorimeter energy (at the high energy limit) in order to provide trigger thresholds with good precision, and the Level-1 Trigger must be robust against calorimeter noise to prevent flooding of the higherlevel trigger and data acquisition system with many useless events. In L1Calo these functions rely on the digital filter implemented in the Preprocessor ASIC.

This paper concentrates on the steps used to determine the optimum digital filter coefficients in L1Calo, and analysis of filter performance.

\section{Digital Filter Implementation}

ATLAS is a general purpose detector consisting of an inner tracking detector, e.m. and hadronic calorimeters, and 


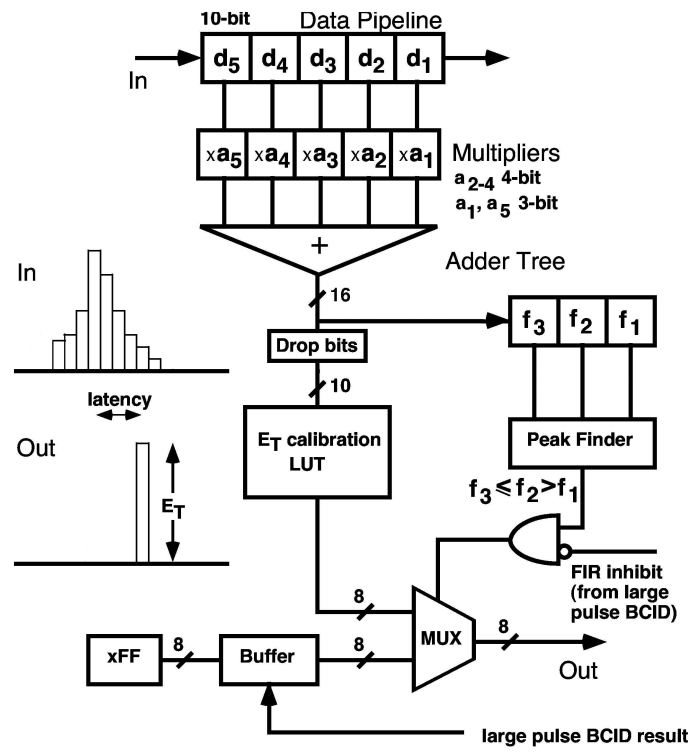

Fig. 1. A diagram showing the implementation of the digital filter and peak-finder as the BCID method for non-saturated pulses. The final energy calibration is implemented in a look-up table.

outer muon chambers. The ATLAS calorimeters have in total $\sim 200,000$ channels. This is too many to be processed by the Level-1 Trigger. Instead, analogue signals are summed transversely and in depth within each calorimeter subsystem into around 7200, approximately projective, trigger towers. The towers are mostly of size $\eta \times \phi \sim 0.1 \times 0.1$. Trigger tower signals are typically much longer than a single bunch crossing. This raises the problem of assigning a pulse to a particular bunch-crossing. The natural choice to make is to choose the peak of the pulse. However, for small signals, noise can distort the shape of the pulse and cause the wrong bunch crossing to be chosen. Conversely, for large, saturated signals there is no clear peak. For saturated signals, a method based on the shape of the leading edge of the pulse is used. [2]

For non-saturated signals (below $\sim 250 \mathrm{GeV}$ ) a combination of a digital filter and a peak-finder is used. The analogue pulse is digitised with ten bit precision and passed through a Finite Impulse Response Filter (FIR Filter). The implementation in the Preprocessor is shown in Figure 1.

Due to both time and chip size limitations, only a simplified filter can be used, limited to five elements, each of which has only four bit precision. This simplification reduces the time and number of logic elements needed to perform the filter calculation, but still gives enough freedom to produce a filter which is tailored for the typical calorimeter signal shape and noise.

The output from the filter is,

$$
f_{\text {output }}=\sum_{i=1}^{5} a_{i} d_{i}
$$

where $a_{i}$ are the filter coefficients and $d_{i}$ are the ADC data samples. Assuming that the pulse shape is independent of energy, then equation 1 can be broken down into its signal, noise and pedestal components,

$$
f_{\text {output }}=E \sum_{i=1}^{5} a_{i} x_{i}+\sum_{i=1}^{5} a_{i} b_{i}+p \sum_{i=1}^{5} a_{i}
$$

where $x_{i}$ describe the normalised signal pulse shape in the absence of noise, $b_{i}$ are the background noise components of the pulse, $E$ is the transverse energy of the pulse and $p$ is the pedestal.

The sixteen bit filter output is passed through a peak finder, which compares the filter output with the outputs from the preceding and following bunch-crossings. The filter output must be greater than preceding value and greater than or equal to the following value to pass the non-saturated BCID requirement.

In parallel, the sixteen bit filter output is fed into a ten bit look-up table (LUT). The LUT simultaneously applies pedestal subtraction, noise cuts and the final transverse energy calibration. The LUT also provides the mechanism for disabling channels, which is done by setting the entire LUT range to output 0 .

For Gaussian white noise, the optimal choice of filter coefficients is the matched filter [3]. A matched filter has coefficients that are chosen to be proportional to the pulse height at each sample. The effect is to sharpen the pulse, improving the signal-to-noise-ratio and the BCID efficiency.

In theory, the best performance is achieved by using a filter matched individually to each tower's signal pulse shape. However, there are several practical limitations. Pulse shapes are not the same for all trigger towers. Relative timing differences and different cable lengths as well as different calorimetry and front-end electronics result in different pulse shapes. Filter coefficients can be set individually for each tower at the cost of increased overall complexity of the system.

The filter coefficients have limited bit precision: four bits unsigned for the central coefficients and four bits signed for the first and last coefficients. To achieve the best match to the pulse shape, the central coefficient should be chosen to be 15 . However, there is an additional complication that the filter's sixteen bit output must be mapped to the ten bit input to the look-up table. This is done by dropping a combination of least significant and most significant bits. In practice, some of the most-significant bits can only ever be used by saturated pulses. A constant value is output for all saturated pulses, and so these bits do not add to the precision of the energy measurement. Therefore, bits that are used only for saturated pulses can be dropped with no loss of precision. In the case that the filter output does overflow ten bits, the maximum value is set. However, usually some least significant bits must also be dropped to reduce the filter output to ten bits, which results in a loss of precision. The number of least-significant bits dropped is chosen so that non-saturated pulses produce a filter output that is within the LUT range after the drop-bits operation.

The best LUT precision is achieved by choosing coefficients such that the entire LUT input range is used for non-saturated pulses only. The LUT precision depends on the filter coefficients, pulse shape and the number of least-significant bits dropped, $n$, 


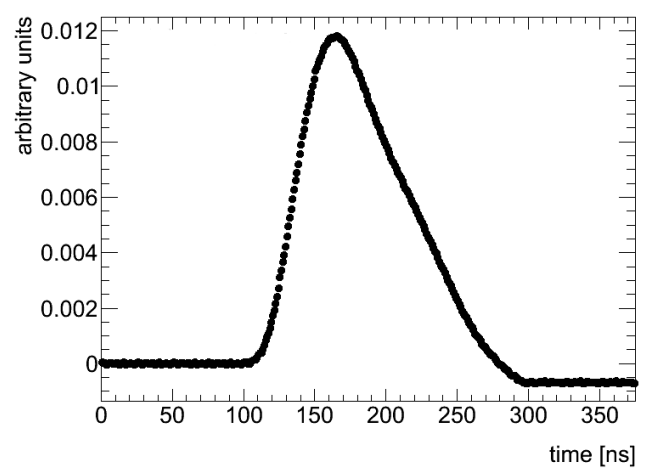

Fig. 2. A typical trigger tower pulse shape reconstructed from a timing scan as explained in section III. A single bunch-crossing is $25 \mathrm{~ns}$. The pulse is many bunch-crossings wide. This pulse is from a LAr trigger tower and shows the long under-shoot following the positive pulse.

$$
\Delta E_{\mathrm{LUT}}=\frac{2^{n}}{\sum_{i=1}^{5} a_{i} x_{i}}
$$

When usage of the LUT range is optimised this gives a precision of $\sim 250 \mathrm{MeV}$. With the wrong choice of filter coefficients and drop-bits settings, it is possible to leave up to half of the LUT range unused, which would result in a precision of $\sim 500 \mathrm{MeV}$. The LUT precision is particularly important for the measurement of small pulses as it defines the precision with which the pedestal subtraction and noise cuts can be applied. In general, in the digital system, it is not possible to simultaneously optimize the LUT range usage and the precision with which the filter may be matched to the pulse shape.

\section{Measurement of Pulse Shapes}

ATLAS uses two different sampling calorimeter technologies: Tile scintillators read out with PMTs, used in the hadronic layer at $|\eta|<1.5$; and LAr ionisation, used across the entire e.m. layer and in the hadronic layer at $|\eta|>1.5$. Different calorimeter technology, front-end electronics and cable lengths can cause large variations in pulse shape throughout the detector.

The L1Calo Preprocessor digitises signals at the LHC bunch-crossing rate. To reconstruct the pulse shape with a finer time interval than the $40 \mathrm{MHz}$ ADC strobe, a timing scan is performed. Calibration pulses are injected into the calorimeter systems and the Preprocessor FADC fine-timing is repeatedly shifted by $1 \mathrm{~ns}$. This allows the pulse shape to be reconstructed with ns precision as shown in Figure 2.

Figure 3 shows the full-width-at-half-maximum of pulses reconstructed from a timing scan. Despite the different technologies used in the hadronic layer, a combination of Tile and LAr calorimetry, pulses are consistently narrower in the hadronic layer than in the e.m. layer. For this reason, when investigating the performance of filters optimised for certain detector regions, it is more appropriate to make a distinction between the e.m. and hadronic layers, than between Tile and LAr calorimeters. Pulses in the forward calorimeters, $|\eta|>3.2$, are significantly narrower than elsewhere in the
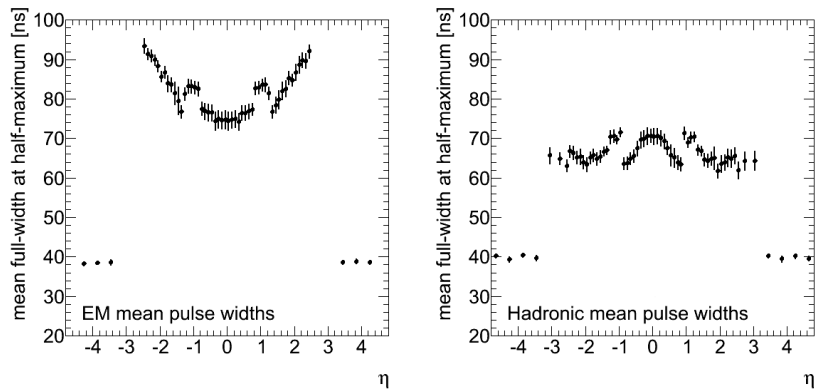

Fig. 3. The mean full-width-at-half-maximum of pulses reconstructed from a timing scan versus $\eta$ shown for the e.m. layer (left) and for the hadronic (right). The bars on the data points show the standard deviation of widths in that $\eta$ bin.

TABLE I

COMMON FILTER COEFFICIENTS

\begin{tabular}{c|c|c} 
Calorimeter Region & Filter Coefficients & \# bits dropped \\
\hline e.m. calorimeters & $1,8,13,10,7$ & 5 \\
\hline hadronic calorimeters & $1,9,15,11,5$ & 5 \\
\hline forward calorimeters & $0,2,13,5,0$ & 4
\end{tabular}

calorimeter, and therefore must also be treated differently to other regions.

\section{Digital Filter Performance}

The filter performance can be accurately simulated using a combination of calibration pulses and noise data. The pulse shapes, as measured from timing scans, were sampled every $25 \mathrm{~ns}$, centred around the peak of the pulse. Pulses from collisions are expected to have shapes approximately equal to the calibration pulse shapes. These digitised pulses were scaled to specific energies and added to real detector noise from events selected with a random trigger. The resulting pulses were used as the input to a simulation of the Preprocessor filter and BCID mechanism, in order to test the performance of different filter configurations. As the filter implementation is entirely digital, its behaviour can be simulated exactly.

Each of the 7168 towers requires 5 FIR coefficients, giving in total 35840 parameters. Optimising the filter on a towerby-tower basis would greatly increase the complexity of the system. This would only be acceptable if it was associated with a significant increase in performance. The performance of the following filters was compared:

1) The matched filter, where the filter was matched individually to each tower's reconstructed pulse shape.

2) A common filter, applied across an entire calorimeter layer. The coefficients used were matched to the median width pulse in the range $|\eta|<0.1$. Low $\eta$ towers were selected as this is where the detector noise is greatest. Detector noise is reduced as $\eta$ increases due to gains applied to convert signals to $E_{\mathrm{T}}$. The sets of coefficients used are shown in Table I.

3) The pass-through filter, where the coefficients for all towers were set to $(0,0,1,0,0)$ and no least-significant bits were dropped. The input pulse is equal to the output pulse and hence this setting is equivalent to using no filter. 


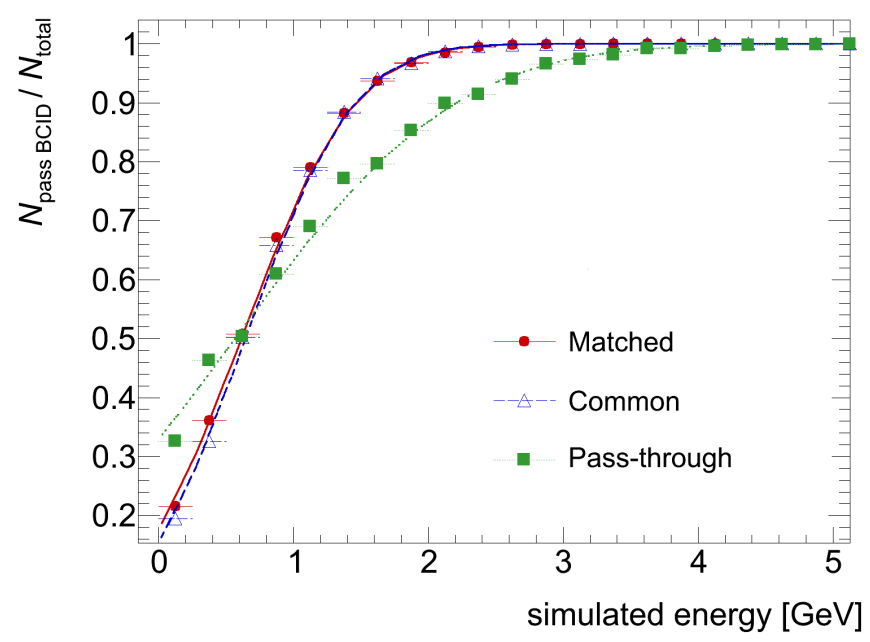

Fig. 4. BCID efficiency turn-on curve comparing Matched, Common and Pass-through filters for a single trigger tower. The fit is given by equation 4 .

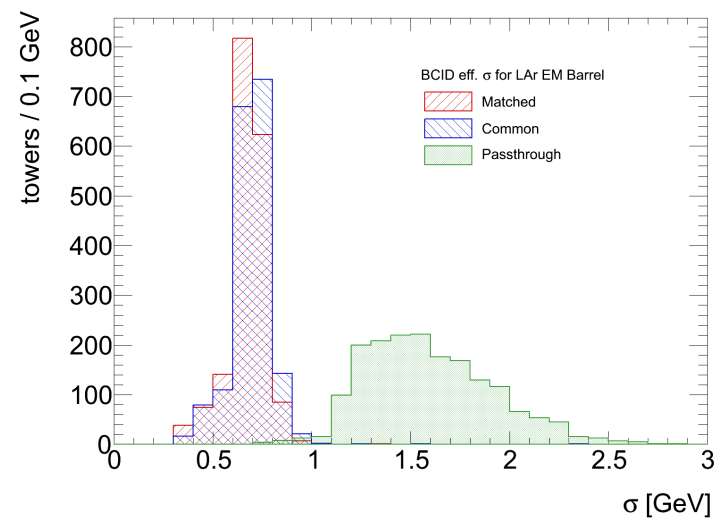

Fig. 5. BCID efficiency turn-on curve $\sigma$, as defined in equation 4 for all towers in the LAr EM barrel. Results for Matched, Common and Pass-through filters are shown.

There are three main measures of performance to compare different sets of filter coefficients: BCID efficiency, noise rejection and energy resolution. Performance results are only shown for towers in the LAr as these towers generally have wider pulses so show greater difference in performance with and without a filter. Absolute performance varies through the calorimeters but the relative performance between the tested filters is consistent.

The efficiency for assigning a pulse to the correct bunchcrossing for a single tower is shown in Figure 4. There is a clear improvement in using the matched or common filters over the pass-through. However, there is little difference between the matched and common filters. The BCID efficiency turn-on curve can be modelled with the equation,

$$
\epsilon(E)=\frac{1}{2}\left(1+\operatorname{erf}\left(\frac{x-\mu}{\sqrt{2 \sigma^{2}}}\right)\right)
$$

where $\epsilon$ is the BCID efficiency, $\mu$ describes the turn-on transverse energy and $\sigma$ describes the width of the turn-on curve. Equation 4 was fitted to the BCID efficiency curves for all towers. The parameter, $\sigma$, is shown for all towers in the

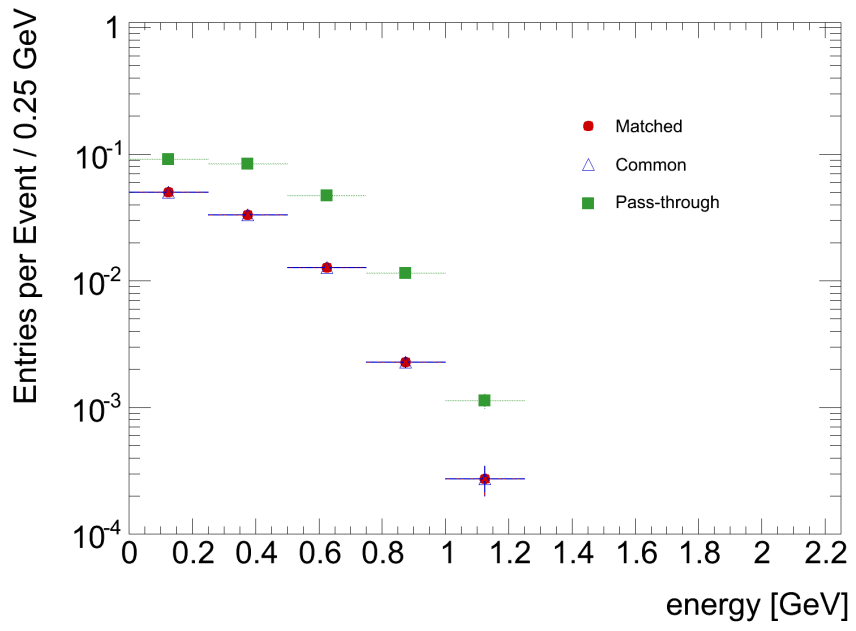

Fig. 6. Probability distribution for noise producing a filter output that passes BCID requirements versus the corresponding $E_{\mathrm{T}}$ for a single tower. Matched, Common and Pass-through filter are shown.

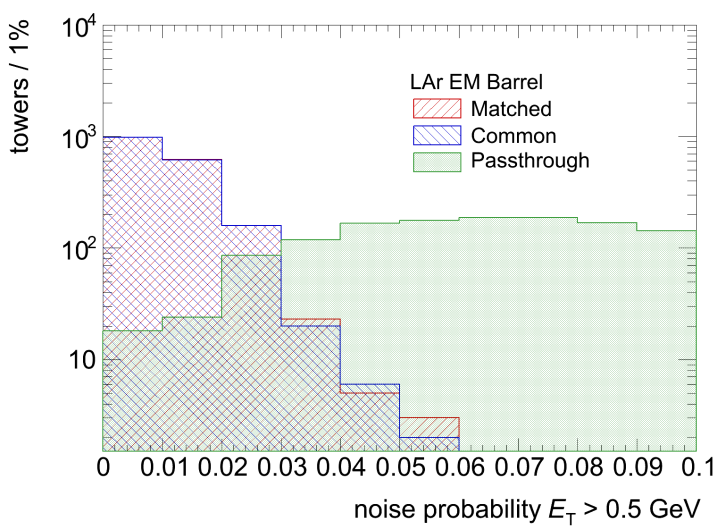

Fig. 7. The probability of a tower's noise producing a filter output with an $E_{\mathrm{T}}$ greater than $0.5 \mathrm{GeV}$ was calculated. The histogram is filled with these probabilities, with an entry for each trigger tower in the LAr EM barrel. Note that this does not equal the probability that a tower would produce a non-zero LUT output that is propagated to the processor systems. Some pulses could be rounded down to $0 \mathrm{GeV}$, and noise cuts are also applied.

e.m. barrel region in Figure 5. These results for are consistent with those shown in the single-tower Figure 4.

If noise fluctuates upwards there is a chance that it will produce a non-zero output from the LUT. By using a filter that includes multiple time slices, there is a tendency for the noise to cancel out, and hence a reduced noise rate. Figure 6 shows the probability distribution of noise producing a filter output with an given $E_{\mathrm{T}}$, for a single tower. Only events passing the BCID criteria are included. There is a clear improvement in using the matched or common filters over the pass-through. However, there is no significant difference between the Matched and Common filters. The distribution shown in figure 6 was integrated from $0.5 \mathrm{GeV}$ to infinity. This gives the probability that noise in that tower would produce a filter output greater than $0.5 \mathrm{GeV}$. Figure 7 shows a histogram filled will the calculated probabilities for all towers in the e.m. barrel region. This represents the worst-case scenario, as this corresponds to all noise pulses that could be rounded 


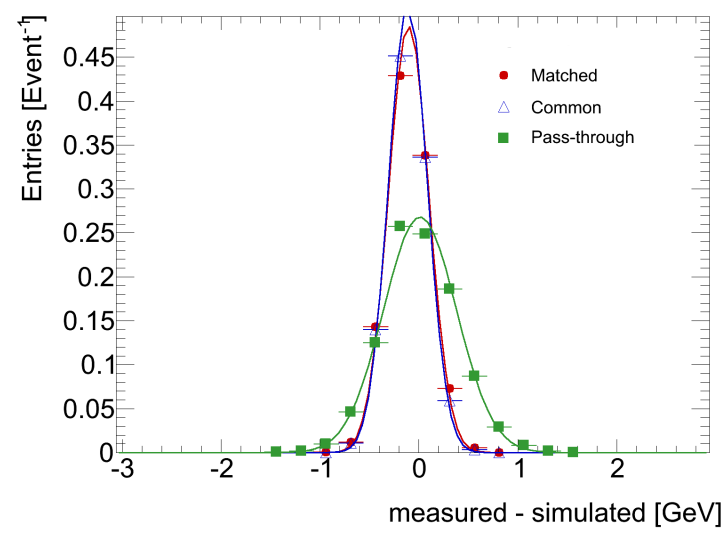

Fig. 8. Difference between simulated $E_{\mathrm{T}}$ and corresponding $E_{\mathrm{T}}$ of the filter output for an individual trigger tower. The Matched, Common and Passthrough filters are shown. A Gaussian is fitted to each distribution.

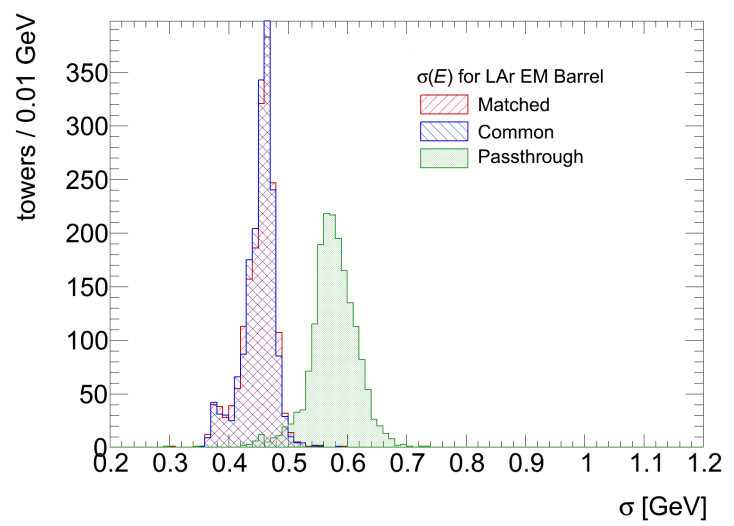

Fig. 9. The $E_{\mathrm{T}}$ resolution for all channels in the LAr EM barrel as determined from a Gaussian fit to the distributions shown in Figure 8.

up to $1 \mathrm{GeV}$ in the LUT table and produce a non-zero output. In practice, noise cuts are applied that drastically reduce the probability of noise producing a non-zero LUT output that is propagated into the processor system. The results for many towers, shown in Figure 7, are consistent with those for a single tower in Figure 6.

Finally, the filter affects the contribution of detector noise to the energy measurement. Figure 8 shows the difference between the $E_{\mathrm{T}}$ measured by L1Calo and the simulated $E_{\mathrm{T}}$. The distribution is narrower when using the matched or common filters compared to the pass-through. However, there is no significant difference between the resolutions of the Matched and Common filters. A Gaussian was fitted to the distributions and the fit widths of all towers in the e.m. barrel region are shown in Figure 9. These results are consistent with those for a single tower in Figure 8.

For all three measures of performance, there is no significant advantage to matching the filter on a tower-by-tower basis. Equivalent performance can be achieved with a common filter applied across an entire calorimeter layer. Therefore, the common filter strategy was adopted for early LHC running.

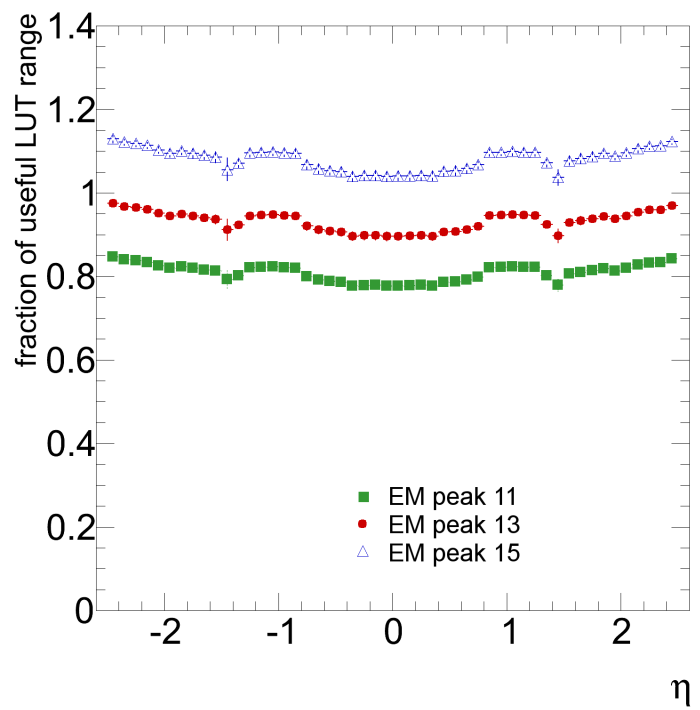

Fig. 10. The fraction of the LUT range that is used by non-saturated pulses in the e.m. layer. Common-type filters are shown scaled to peak coefficients of 15,13 and 11 . The meaning of these values are explained in the text.

\section{Optimising USAGE OF THE LUT DyNAMiC RANGE}

There are two places within the Preprocessor system where saturation can occur. Firstly, the input analogue pulses are digitised with ten bit precision. Analogue gains are applied to calibrate the input scale to 4 counts per $\mathrm{GeV}$ of $E_{\mathrm{T}}$. There is a nominal pedestal of 32 counts. Hence the ADC saturates at an $E_{\mathrm{T}}$ of approximately $248 \mathrm{GeV}$. Secondly, the input to the LUT is only ten bits wide. In general, the energy scales before and after applying the filter are different. Consequently, the corresponding saturation energies are different.

To achieve the best precision for matching the pulse shapes the filter must have a central coefficient value of 15 . However, by rescaling the filter to different peak heights, it is possible to control the LUT saturation energy. Figure 10 shows the filter output for 3 different scalings of the Common e.m. filter as a fraction of the LUT input range.

The ideal situation is one where the ADC and LUT saturation energies are the same. When the e.m. filter is scaled to a peak of 15, the ADC saturation energy is greater than the LUT saturation energy. In this case, the LUT cannot output the full range of pulse energies that can be described by the raw ADC pulse. When the e.m. filter is scaled to a peak of 11, the ADC saturation energy is less than the LUT saturation energy. In this case, up-to $20 \%$ of the LUT's dynamic range is wasted on saturated pulses. This reduces the granularity of LUT counts and so reduces the precision with which pedestal subtraction and noise cuts can be applied. By scaling the e.m. filter to a peak of 13, most of the LUT range is used effectively. Analysis of these scaled filters showed no significant difference in performance.

Importantly, Figure 10 also shows that a Common filter is capable of providing reasonably uniform saturation energies across the detector; it is not necessary to optimise the LUT range usage on a tower-by-tower basis. 


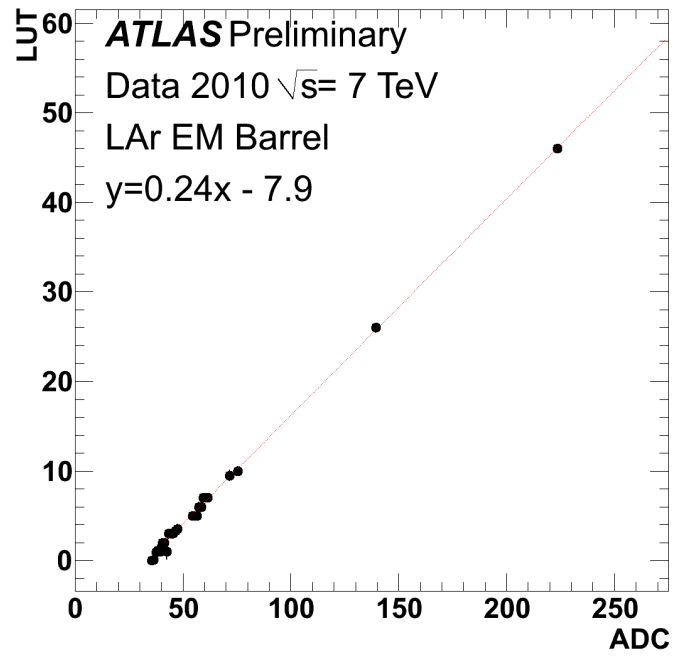

Fig. 11. Correlation between ADC peak slice and LUT output for a single tower from collisions pulses. The offset from the origin is due to the pedestal. A straight line is fit to the data points and the fit gradient is 0.24 , close to the expected value of 0.25 .

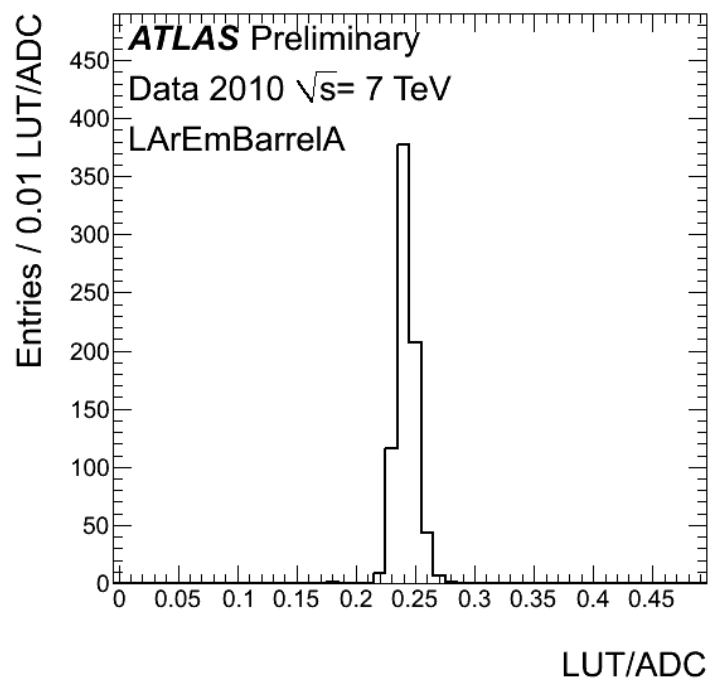

Fig. 12. Fit gradients of ADC-LUT correlation for all channels in the LAr Barrel A-side.

\section{LUT $E_{\mathrm{T}}$ CALIBRATION}

The output from the filter is passed into the LUT where pedestal subtraction, noise cuts and the final $E_{\mathrm{T}}$ calibration are applied. For early running, the LUT table $E_{\mathrm{T}}$ calibration was based on a simple linear slope set to convert the filter output back to the input $E_{\mathrm{T}}$ scale. The initial LUT $E_{\mathrm{T}}$ calibration was based on measurements of calibration pulses. However, timing and pulse shapes could be different and lead to an incorrect energy measurement. The LUT $E_{\mathrm{T}}$ calibration was checked by measuring the relationship between the peak ADC sample of a pulse and the LUT output for collision pulses. The ADC input is ten bits wide and the LUT output is only eight bits wide. Hence, if the LUT is perfectly calibrated for collisions then the gradient should be $\frac{1}{4}$. Figure 11 shows the correlation between ADC peak sample and LUT output for a single trigger tower. The gradients were extracted from a linear fit to the data and are shown in Figure 12 for all towers in the e.m. barrel on the A-side of the detector. Most channels are within a few percent of the optimum value. The calibration based on test pulses is already working well for pulses from collisions, though further improvements are possible. Future calibration will be based on physics objects reconstructed offline.

\section{SUMMARY}

The L1Calo Preprocessor's digital filter provides significant improvements in bunch-crossing identification of small signals, noise rejection and energy resolution. With the limited precision available in the filter coefficients, a common filter provides performance equivalent to that of filters tuned on a channel-by-channel basis, but with vastly reduced complexity in the system. Furthermore, the filter can be optimised to make best use of the look-up table range, at the expense of the precision match to the pulse shape, with no loss in performance.

Cross-checks of the initial LUT $E_{\mathrm{T}}$ calibration show that it is already working well for pulses in early LHC beam collision data, though further calibration is required.

Based on the studies presented in this paper, a set of Common filters applied across each calorimeter layer, with coefficients scaled to make best use of the LUT range, were adopted for 2009-10 running. The coefficients used are shown in Table I.

\section{ACKNOWLEDGMENT}

We are greatly indebted to all CERN's departments and to the LHC project for their immense efforts not only in building the LHC, but also for their direct contributions to the construction and installation of the ATLAS detector and its infrastructure. All our congratulations go to the LHC operation team for the superb performance during this initial data-taking period. We acknowledge equally warmly all our technical colleagues in the collaborating Institutions without whom the ATLAS detector could not have been built. Furthermore we are grateful to all the funding agencies which supported generously the construction and the commissioning of the ATLAS detector and also provided the computing infrastructure.

The ATLAS detector design and construction has taken about fifteen years, and our thoughts are with all our colleagues who sadly could not see its final realisation.

\section{REFERENCES}

[1] R Achenbach et al, "The ATLAS Level-1 Calorimeter Trigger", JINST 3 P03001 (2008)

[2] U. Pfeiffer and W. Htzel, "Bunch-Crossing Identification for saturated calorimeter signals", ATLAS Note ATL-DAQ-99-009 (1999)

[3] J. Garvey and D. Rees, "Bunch Crossing Identification for the ATLAS Level-1 Calorimeter Trigger", ATLAS Internal Note ATL-DAQ-96-051 (1996) 